\title{
THE LACK OF HALO ULTRALUMINOUS X-RAY SOURCES
}

\author{
Douglas A. SWARTz ${ }^{1}$ \\ Submitted to Astrophysical Journal Letters
}

\begin{abstract}
The premise that Ultraluminous X-ray sources (ULXs) exist beyond the optical extent of nearby galaxies is investigated. A published catalog containing 41 ULX candidates located between 1 and $\sim 3$ times the standard $D_{25}$ isophotal radius of their putative host galaxies is examined. Twenty-one of these sources have spectroscopically-confirmed distances. All 21 are background objects giving a 95\% probability that at least 37 of the 41 candidates are background sources. Thirty-nine of the 41 sources have X-ray-to-optical flux ratios, $-1.6<\log \left(F_{\mathrm{X}} / F_{\mathrm{O}}\right)<+1.3$, consistent with those of background active galactic nuclei. (The remaining two are not detected in optical to a weak limit of $m_{B} \sim 21.5 \mathrm{mag}$ corresponding to $\log \left(F_{\mathrm{X}} / F_{\mathrm{O}}\right) \gtrsim 1.6$. $)$ The uniform spatial distribution of the sample is also consistent with a background population. This evidence suggests that ULXs rarely, if at all, exist beyond the distribution of luminous matter in nearby galaxies and, as a consequence, there is no correlation between the population of ULXs and halo objects such as old globular clusters or Pop III remnants.
\end{abstract}

Subject headings: galaxies: general — galaxies: halos — X-rays: galaxies — X-rays: general

\section{INTRODUCTION}

Ultraluminous X-ray sources (ULXs) are point-like nonnuclear sources in nearby galaxies with apparent X-ray luminosities $L_{\mathrm{X}} \gtrsim 10^{39} \mathrm{ergs} \mathrm{s}^{-1}$. Their nature is still a mystery: if they are accreting sources at the distance of their putative host galaxies, then their high luminosities require either beamed emission geometries, or super-Eddington emission rates, or accretion onto compact objects more massive than predicted by stellar evolution models. See Fabbiano (2006) for a review of ULX theory, observation, and their importance in studies of extragalactic X-ray source populations.

Distances to candidate ULXs can, in principal, be determined spectroscopically if they have bright optical counterparts. Because spectroscopy requires high contrast between the optical counterpart and the underlying galaxy light, most spectroscopic measurements made to date have been of bright counterparts located in the low surface brightness regions at relatively large distances from the centers of their putative host galaxies. The fact that all these spectroscopically-examined objects have been confirmed $^{2}$ to be background sources instead of true ULXs suggests ULXs may not exist beyond the luminous regions of galaxies and hence that there is no association between ULXs and halo objects.

\section{PROPERTIES OF A ULX CANDIDATE SAMPLE}

To investigate the merits of this conjecture, a sample of ULX candidates located in the outlying regions of their host galaxies is examined. The ULX candidate catalog of Colbert \& Ptak (2002) is chosen for this purpose because (1) it includes candidates out to twice the optical radius of the target galaxies and (2) López-Corredoira \& Gutiérrez (2006) have recently compiled the known optical properties of all the ULX candidates in this catalog. The Colbert \& Ptak (2002) catalog is based on positional coincidences be- tween point-like X-ray sources in RoSat/HRI images and galaxies listed in the Third Reference Catalogue of Bright Galaxies (RC3; de Vaucouleurs et al. 1991). The ULX catalog tabulates celestial coordinates and X-ray luminosity estimates for 87 ULX candidates detected in 54 galaxy fields (see Colbert \& Ptak 2002; Ptak \& Colbert 2004 for details).

The optical extent of a galaxy is here defined to be the ellipse enclosing the isophote at surface brightness $25 \mathrm{~B}$ mag $\operatorname{arcsec}^{-2}$. The major axis diameter $\left(D_{25}\right)$, major-to-minor axis ratio, and position angle of the ellipses are taken from the RC3. The deprojected angular distance of each ULX candidate from the center of the host galaxy can be expressed as the fraction, $f_{\mathrm{D} 25}$, of the angular distance to the $D_{25}$ isophote along the radius from the galaxy center to the ULX candidate. Sources outside the optical extent of the host galaxies are defined as those with $f_{\mathrm{D} 25}>1$. Fortyone of the 87 ULX candidates listed in the Colbert \& Ptak (2002) catalog have $f_{\mathrm{D} 25}>1$. Note that the $D_{25}$ isophote is a reasonable demarcation, on average, between the luminous bulge-disk regions of a galaxy and its dark halo while the specific division at $f_{\mathrm{D} 25}=1$ (and the approximation of the $D_{25}$ isophote as an ellipse) is merely a convenient expedient that could be varied somewhat without altering the results to follow.

A histogram of the distribution of $f_{\mathrm{D} 25}$ for all 87 ULX candidates is displayed in Figure 1. The ordinate is the number, $N\left(f_{\mathrm{D} 25}\right)$, of ULX candidates per unit $f_{\mathrm{D} 25}$ area. Since the angular size, $D_{25}$, varies from galaxy to galaxy, the physical scale represented by unit $f_{\mathrm{D} 25}$ area also varies among the ensemble of sources. However, it does correctly weight each bin such that $N\left(f_{\mathrm{D} 25}\right)$ is independent of $f_{\mathrm{D} 25}$ for a uniform spatial distribution of sources. The distribution of ULX candidates in this space is flat in the range $1<f_{\mathrm{D} 25}<2$ according to Figure 1 . For $f_{\mathrm{D} 25}<1$, the distribution of ULX candidates increases inward to a maximum

\footnotetext{
${ }^{1}$ Universities Space Research Association, NASA Marshall Space Flight Center, VP62, Huntsville, AL, USA

${ }^{2}$ Confirmation formally applies only to the optical source but, under these circumstances, the probability of a chance positional coincidence is small and it is likely the $\mathrm{X}$-ray and optical sources are physically related.
} 
at $f_{\mathrm{D} 25}=0$. This is the same trend reported previously for $f_{\mathrm{D} 25} \leq 1$ ULX candidates (cf. Figure 12 of Swartz et al. 2004; Figure 16 of Liu et al. 2006; see also Irwin et al. 2004) and roughly follows the distribution of optical emission from the host galaxies. Beyond $f_{\mathrm{D} 25}=2$, the distribution declines because of incomplete sampling: Colbert \& Ptak (2002) considered sources within a circle of radius $r=D_{25}$ about the galaxy centers so $f_{\mathrm{D} 25}>2$ occurs only in a subset of azimuthal angles symmetric about the $D_{25}$ ellipse's minor axis (and favors contributions from high-inclination spiral galaxies with highly eccentric $D_{25}$ ellipses).

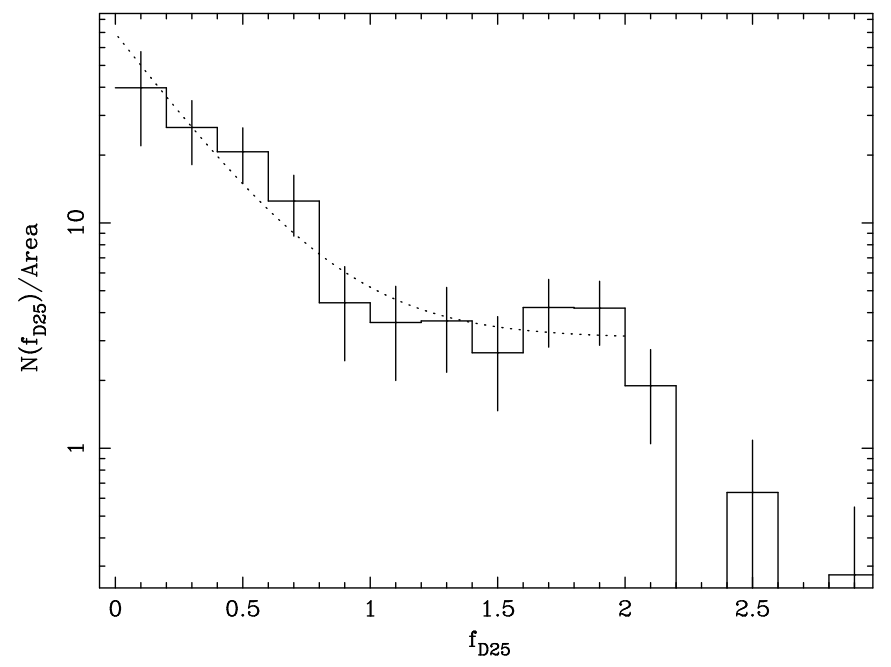

FIG. 1. - Spatial distribution of 87 ULX candidates from the catalog of Colbert \& Ptak (2002). The abscissa is the distance of the ULX candidate from the center of its host galaxy in units of the $D_{25}$ isophotal diameter and the ordinate is the number of ULX candidates per unit $f_{\mathrm{D} 25}$ area. The dotted curve is the best-fit constant plus exponential to the data on the range $0<f_{\mathrm{D} 25}<2$.

A more quantitative statement can be made by fitting a constant plus an exponential function to the data on the range $0<f_{\mathrm{D} 25}<2$. Integrating the best-fit function, $N\left(f_{\mathrm{D} 25}\right)=3.1+66.3 \exp \left(-f_{\mathrm{D} 25} / 0.3\right)\left(\chi^{2}=4.7\right.$ for 7 dof), over the range $1<f_{\mathrm{D} 25}<2$ gives a contribution of 29.2 sources from the uniform distribution (represented by the constant term) and 4.6 sources from the exponential distribution. This implies $86 \%$ of the ULX candidates beyond $f_{\mathrm{D} 25}=1$ are part of the uniform spatial distribution of background sources. (There are a total of 33 sources in the sample on this range and another 8 with $f_{\mathrm{D} 25}>2$.) Within $f_{\mathrm{D} 25}=1$, the constant term contributes 9.7 sources and the exponential 30.0 sources implying only $24 \%$ are background. This is consistent with simply assuming background sources are uniformly distributed; from the ratio of areas, 13.7 background sources (30\%) are expected within $f_{\mathrm{D} 25}=1$ if all 41 sources beyond $f_{\mathrm{D} 25}=1$ are background.

López-Corredoira \& Gutiérrez (2006) have tabulated some optical properties of the ULX candidates in the Colbert \& Ptak (2002) catalog. Here, only the 41 sources with $f_{\mathrm{D} 25}>1$ are considered. Figure 2 displays their location in an X-ray-optical flux-flux diagram (the X-ray fluxes are also from the López-Corredoira \& Gutiérrez tabulation). X-ray-selected background sources (mostly AGN) detected in deep field (e.g., Brandt \& Hasinger 2005) and in serendipitous wide-field (e.g. Green et al. 2004) Xray surveys typically have $\mathrm{X}$-ray-to-optical flux ratios in the range $-1<\log \left(F_{\mathrm{X}} / F_{\mathrm{O}}\right)<+1$ with a mean around $\log \left(F_{\mathrm{X}} / F_{\mathrm{O}}\right) \sim 0$. Lines depicting $\log \left(F_{\mathrm{X}} / F_{\mathrm{O}}\right)=-1,0$, +1 are shown ${ }^{3}$ in Figure 2. The $f_{\mathrm{D} 25}>1$ ULX candidates positionally-coincident with optically-bright counterparts occupy the same range in flux-flux space as do the background X-ray population.

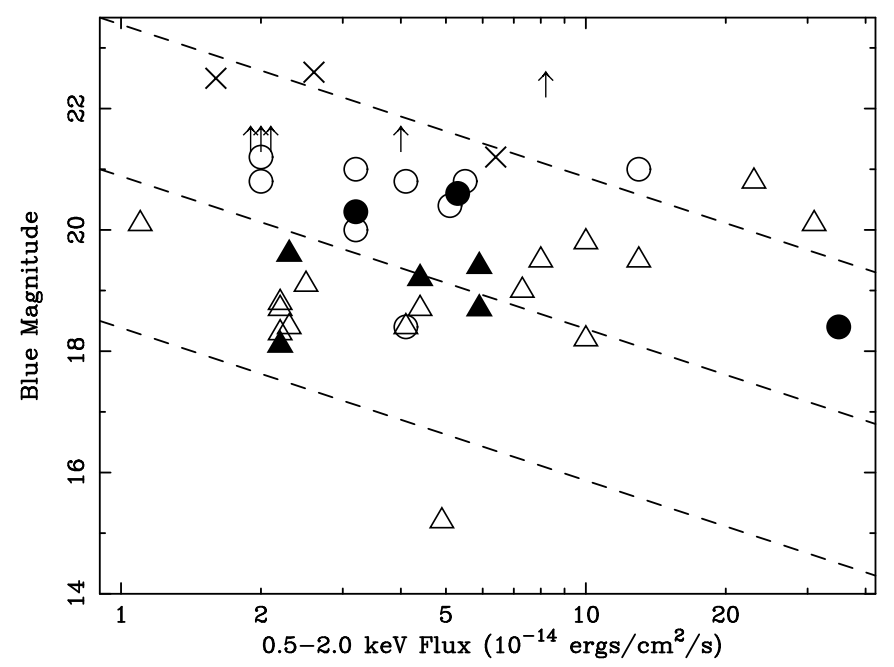

FIG. 2.- $B$-band magnitude versus $0.5-2.0 \mathrm{keV} \mathrm{X}$-ray flux for the 41 ULX candidates with $f_{\mathrm{D} 25}>1$. Data from López-Corredoira \& Gutiérrez (2006). Triangles represent objects with spectroscopic redshifts. Xs denote sources cataloged in the Sloan Digital Sky Survey but listed only as upper limits in López-Corredoira \& Gutiérrez. Arrows denote X-ray sources not detected in optical data and are located at their $B$ magnitude limit. Open symbols represent sources in the field of elliptical galaxies and filled symbols represent those in spiral galaxy fields. Dashed lines indicate constant $\log \left(F_{\mathrm{X}} / F_{\mathrm{O}}\right)=-1,0,+1$.

Twenty-one of these sources have had spectroscopic redshifts measured. These are designated by triangles in Figure 2. They tend naturally to be the optically-brightest sources. The redshifts of all 21 of these sources confirm they are background objects. Assuming this is an unbiased sample and taking the distribution of possibilities to be bimodal (they either are or are not background objects), then there is a $95 \%$ probability that $90 \%$ or more of the population of $f_{\mathrm{D} 25}>1$ ULX candidates are background objects. (Note that, since the spectroscopic sample is not randomly selected but favors optically-bright objects, the possibility remains that some or even all of the optically-faint ULX candidates are indeed an independent population unrelated to the confirmed background sources, though they are still distributed uniformly and have $F_{\mathrm{X}} / F_{\mathrm{O}}$ ratios consistent with background objects.)

López-Corredoira \& Gutiérrez (2006) reported potential optical counterparts for all but 8 of the 41 ULX candidates beyond $f_{\mathrm{D} 25}=1$. Three of these 8 are visible in Sloan Digital Sky Survey (SDSS) Data Release 5 images and designated with Xs in Figure 2 (using $m_{B}=g+0.31(g-r)+0.23$ to convert from $g$ and $r$ SDSS magnitudes). They are all classified by SDSS as galaxies. Only upper limits based on Digitized Sky Survey (DSS) blue images or SDSS are available for the remaining five ULX candidates. These

\footnotetext{
${ }^{3}$ The Johnson I or R bands or their equivalent are typically used for $F_{\mathrm{O}}$ instead of B as quoted in López-Corredoira \& Gutiérrez (2006) and used here.
} 
are marked with arrows at the positions of their X-ray flux and limiting optical magnitudes. Three of these 5 sources are X-ray faint and fall comfortably below the line $\log \left(F_{\mathrm{X}} / F_{\mathrm{O}}\right)=+1$; consistent with background sources. This leaves two ULX candidates beyond $f_{\mathrm{D} 25}=1$ that cannot readily be interpreted as background objects based on either measured redshifts or on the known $F_{\mathrm{X}} / F_{\mathrm{O}}$ trend among background objects. The weaker source, at $F_{\mathrm{X}}=4 \times 10^{-14} \mathrm{ergs} \mathrm{cm}^{-2} \mathrm{~s}^{-1}$, is designated IXO $30 \mathrm{in}$ Colbert \& Ptak (2002). Bianchi et al. (2005) reported an Fe K $\alpha$ emission line in the XMM-Newton spectrum of this source and that therefore it is likely a foreground Cataclysmic Variable rather than a ULX. Note that low-mass and even high-mass X-ray binaries are expected to have very high $F_{\mathrm{X}} / F_{\mathrm{O}}$ ratios. For example, the optical counterpart to the moderately-luminous $\left(L_{\mathrm{X}} \sim 3 \times 10^{39} \mathrm{ergs} \mathrm{s}^{-1}\right)$ ULX designated X6 (Fabbiano 1988) in the nearby galaxy NGC 3031 is an O9 - B1 (Swartz et al. 2003) or B8 V (Liu et al. 2002) high-mass star with $B \sim 24$ giving $F_{\mathrm{X}} / F_{\mathrm{O}} \sim 4$.

Figure 2 also shows there are no differences in the distribution of optical or X-ray fluxes of sources in the fields near elliptical compared to spiral galaxies. This is expected if the $f_{\mathrm{D} 25}>1$ sources are background objects unrelated to the putative host galaxies.

Finally, if these $f_{\mathrm{D} 25}>1$ sources are background objects, then their number-flux relation should be consistent with that of the resolved cosmic X-ray background. To make such a comparison requires knowledge of the angular area and the (observation-specific) sensitivity of each X-ray galaxy field in the Colbert \& Ptak (2002) sample including the many observed galaxies that completely lack ULX candidates. While this level of detail is not readily available, Ptak \& Colbert (2004) have shown that the number of galaxies in the sample of Colbert \& Ptak (2002) with ULX candidates above a given luminosity limit is identical for both the $r=D_{25}$ search radius used by Colbert \& Ptak (2002) and for the more restrictive $r=0.5 D_{25}$ radius once the expected background contributions are accounted for. In other words, the number of ULX candidates per unit area and per unit flux detected beyond $f_{\mathrm{D} 25} \sim 1$ is consistent with the known distribution of background sources.

\section{DISCUSSION}

For the sample analysed here, the overwhelming majority, and perhaps all, of the ULX candidates located beyond the optical extent of their host galaxies are unrelated background objects. This, in itself, is not remarkable since it must be true when $f_{\mathrm{D} 25} \gg 1$. What is of interest is the strong distinction between sources inside and outside of $f_{\mathrm{D} 25} \sim 1$. The physical processes that favor production of ULXs within the optical extent of a galaxy must operate rarely, if ever, in galactic halos. Since the most relevant $\mathrm{X}$-ray emission mechanism here is accretion onto a compact object, the lack of halo ULXs means either compact objects are rare or suitable accretion reservoirs are absent in galactic halos. What are the implications of this for ULX models?

There are two classes of object that have been recognized as potential sources of halo compact objects and in particular as candidate ULXs. These are the remnants of zero-metallicity Population III stars and the (possibly massive) black holes nurtured in the cores of globular clusters.

Remnants of the first generation of stars may be massive black holes and many could exist in galactic halos (Madau \& Rees 2001; Islam et al. 2004a). In order to appear as ULXs, these remnants must be accreting at high rates - through a thin disk instead of by Bondi-Hoyle accretion (King et al. 2001) - from the interstellar medium or from their own bound relic minihalos. Their large masses, ¿300 $\mathrm{M}_{\odot}$, imply they should be strong UV/optical sources with $\log \left(F_{\mathrm{X}} / F_{\mathrm{O}}\right) \ll 1$ (Islam et al. 2004b; Volonteri \& Perna 2005). There are no ULX candidates in the present sample that meet these conditions.

Globular clusters are known to host X-ray binaries; in fact, dynamical interactions in the dense cluster environment enhances close binary formation. ULXs in globular clusters would, in general, have $\log \left(F_{\mathrm{X}} / F_{\mathrm{O}}\right) \gtrsim 2$ even for the fainter X-ray sources (e.g., Kundu et al. 2002; Sarazin et al. 2003). But this is close enough to the optically-faint end of the observed range for $f_{\mathrm{D} 25}>1$ objects that a globular cluster host cannot be completely excluded from the present sample.

The importance of these two ULX scenarios is that Pop III remnants and the dense stellar environments of globular clusters are two potential sites for the elusive intermediate-mass black holes sought to fill the gap between stellar-mass and supermassive black holes (van der Marel 2004). In Pop III stars, these form from direct collapse of massive stars. In globular clusters, they may form through the gradual accrual of mass over the long lifetime of the cluster, primarily in the form of accreted black holes (Miller \& Hamilton 2002) or gas accretion (Kawakatu \& Umemura 2005), onto a seed black hole of initial mass large enough to prevent ejection from the cluster through recoil. Alternatively, they may form at the birth of the cluster through runaway stellar or proto-stellar mergers (Portegies Zwart et al. 2004). (It is unclear if these latter clusters survive to become old globular clusters in galactic halos or if their compact objects would appear as ULXs at this late evolutionary stage). This work has shown, whether such objects exist or not, they rarely, if ever, appear as ULXs.

I thank the participants of the informal X-ray Aficionados meetings for motivating discussions and keen insights; in particular S. O'Dell, M. Finger, and A. Tennant. I also thank R. Soria, A. Tennant, and the anonymous referee for helpful comments on the manuscript.

\section{REFERENCES}

Bianchi S., Miniutti, G., Fabian, A. C., Iwasawa, K. 2005, MNRAS, 360, 380

Brandt, W. N., \& Hasinger, G. 2005, ARA\&A, 43, 827

Colbert, E. J. M., \& Ptak, A. F. 2002, ApJS, 143, 25 de Vaucouleurs G., de Vaucouleurs A., Corwin Jr. H. G., Buta R. J., Paturel G., \& Fouque P. 1991, Third Reference Catalogue of Bright Galaxies (New York: Springer-Verlag)

Fabbiano, G. 1988, ApJ, 325, 544

Fabbiano, G. 2006, ARA\&A, 44, 323

Green, P. J., et al. 2004, ApJS, 150, 43 
Irwin, J. A., Bregman, J. N., \& Athey, A. E. 2004, ApJ, 601, L143

Islam, R. R., Taylor, J. E., \& Silk, J. 2004a, MNRAS, 354, 427

Islam, R. R., Taylor, J. E., \& Silk, J. 2004b, MNRAS, 354, 443

Kawakatu, N., \& Umemura, M. 2005, ApJ, 628, 721

King, A. R., Davies, M. B., Ward, M. J., Fabbiano, G., \& Elvis, M. 2001, ApJ, 552, L109

Kundu, A., Maccarone, T. J., \& Zepf, S. E. 2002, ApJ, 574, L5

López-Corredoira, M. \& Gutiérrez, C. M. 2006, A\&A, 454, 77

Liu, J. -F., Bregman, J. N. \& Seitzer, P. 2002, ApJ, 580, L31

Liu, J. -F., Bregman, J. N. \& Irwin, J. 2006, ApJ, 642, 171

Miller, M. C., \& Hamilton, D. P. 2002, MNRAS, 330, 232

Madau, P., \& Rees, M. J. 2001, ApJ, 551, L27
Portegies Zwart, S. F., Baumgardt, H., Hut, P., Makino, J., McMillan, S. L. W. 2004, Nature, 428, 724

Ptak, A. \& Colbert, E. 2004, ApJ, 606, 291

Sarazin, C., Kundu, A., Irwin, J. A., Sivakoff, G. R., Blanton, E. L., Randall, S. W. 2003, ApJ, 595, 743

Swartz, D. A., Ghosh, K. K., McCollough, M. L., Pannuti, T. G., Tennant, A. F., \& Wu, K. 2003, ApJS, 144, 213

Swartz, D. A., Ghosh, K. K., Tennant, A. F., \& Wu, K. 2004, ApJS, 154,519

van der Marel, R.P. 2004, in Coevolution of Black Holes and Galaxies, ed. L.C. Ho (Cambridge:CUP), 37

Volonteri, M. \& Perna, R. 2005, MNRAS, 358, 913 